\title{
A SYSTEMATIC OVERVIEW OF ABDOMINAL AORTIC ANEURYSM (AAA)
}

\author{
Gerardo AK Laksono ${ }^{1)}$, Paul L Tahalele ${ }^{2)}$
}

\begin{abstract}
An abdominal aortic aneurysm (AAA) is defined as an abnormal dilatation of the abdominal aorta more than $50 \%$ of its diameter. The aortic wall continues to weaken and become unable to hold the forces of the luminal blood pressure, resulting in progressive dilatation and rupture. The rupture of the aneurysm wall is influenced by several factors, such as the aneurysm size, expansion rate, continued smoking, and persistent hypertension. The AAA is a common degenerative condition with high mortality in older people. The AAA increases these last two decades. It is occurring in $7-8 \%$ of men at the age of over 65 years old. The disease prevalence is six times higher in men than in women. It is also increasing dramatically by the presence of the following factors: age older than 60 years old, smoking, hypertension, and ethnicity. Repair of large or symptomatic AAA by open surgery or endovascular is recommended, but not significant in the small aneurysm.
\end{abstract}

\footnotetext{
${ }^{1)}$ Student of Faculty of Medicine Widya Mandala Catholic University Surabaya, Kalisari Selatan 1 Surabaya Email : Gerardoagung@gmail.com

${ }^{2)}$ Surgery Department Faculty of Medicine Widya Mandala Catholic University Surabaya, Kalisari Selatan 1 Surabaya
} 


\section{TERMINOLOGY, EPIDEMIOLOGY AND RISK FACTORS}

Derived from the Greek word, aneurysm describing 'a widening.' Abdominal aortic aneurysm itself defined as an abnormal dilatation of the abdominal aorta more than $50 \%$ of its diameter ${ }^{1}$. The dilatation affects the three layers of the vascular tunic; otherwise, the dilatation is called a pseudo-aneurysm. Although aneurysm represents dilatation in all layers of the vessel wall, AAAs developed after the degeneration of media. The degeneration of media leads to the widening of the vessel lumen and loss of structural integrity of the aorta ${ }^{2}$. Most aneurysms are fusiform, where the whole circumference of the artery is affected. Saccular aneurysm termed when aneurysm includes only part of the circumference. An inflammatory aneurysm is characterized by extensive perianeurysmal and retroperitoneal fibrosis and dense adhesions to the adjacent abdominal organs. Aneurysm involving the renal Ostia (infrarenal, suprarenal aorta) are also included under this term ${ }^{3}$.

\section{Population screening stud ies}

indicate that the prevalence of abdominal aortic aneurysm (AAA) increases these last two decades. It is occurring in $7-8 \%$ of men at the age of over 65 years old. The disease prevalence is six times higher in men than in women. AAA is a significant cause of premature death worldwide. Recent estimates suggest an annual death rate due to aortic aneurysm of 2.8/100.000 (>20 million). They are representing a $12 \%$ increase in the last 20 years. In the United States, the AAA becomes a leading cause of death, nearing just less than 5,000 deaths due to the most dreaded complication, rupture ${ }^{3-5}$.

There are many causes of aneurysmal dilatation, but few abdominal aortic aneurysms are the direct consequence of specific such as trauma, acute infection such as salmonellosis and brucellosis, chronic infection such as tuberculosis, inflammatory disease, and connective tissue disorders such as Marfan syndrome and Ehlers-Danlos type $\mathrm{IV}^{1,2,6}$. There are many risk factors associated with AAA include hypertension, vascular disease, atherosclerosis, age, sex, ethnicity, genetic, and smoking ${ }^{7}$. The risk of AAA increases after 60 years old. An aneurysm that has a diameter of more than $4 \mathrm{~cm}$ presents in $1 \%$ of men between 55 and 64 years old. AAA also found to occur more frequently in white people than in black people. Atherosclerosis becomes the most common causative factor for AAA. Patients with AAAs frequently have atherosclerosis, some studies showing the association of coronary heart disease and 
peripheral atherosclerosis. The most compelling argument for the causative role of atherosclerosis in AAA is arterial remodeling. Hypertension has been considered a potential risk factor for AAA. A meta-analysis found a $66 \%$ higher risk of AAA in hypertensive patients compared to non-hypertensive patients and a $14 \%$ and $28 \%$ increase in the risk of the disease for every 20 and 10 $\mathrm{mmHg}$ rise in SBP and DBP, respectively. Smoking also found to be a significant risk of AAA formation. A positive dose-response relationship was observed between the increasing number of cigarettes smoked per day, and packyears smoked and the risk of abdominal aortic aneurysm. Family history is also a risk factor for all types of aortic aneurysms. Range of further mutations has been identified in genes associated with aortic aneurysms such as TGF- $\beta$ receptor type I and II, myosin-11, and alpha two actin and aortic smooth muscle ${ }^{4,8,9}$.

\section{PATHOGENESIS}

Elastic fibers and fibrillar collagens are the main determinants of the mechanical properties of the aorta. Elastic fibers are highly elastic and stretch in response to tension. A network of elastic fibers is responsible for the viscoelastic properties of the vascular formed by elastin and other forms of proteins. Elastic fibers associated with smooth muscle cells are most abundant in the media of the aortic wall ${ }^{8,10}$. Elastin that forming the elastic fibers has a vital role in the load-bearing element of the aorta. Elastin fragmentation and degeneration are observed in the aneurysm wall. Collagen is also a significant component of the media and surrounding fibrous adventitia 6,11,12. Biomechanical and clinical studies demonstrate that the mechanical strength of the vascular walls relies on the structural collagen network in the media and adventitia. An increasing number of collagen degradation without adequate collagen synthesis and deposition is held responsible for the growth of AAA. Two specific types of collagen (type I and III) provide tensile strength and help maintain the integrity of the wall of the vascular structure that is highly resistant toward proteolysis ${ }^{2,8,10,13}$. A study by Dobrin and Mrkvicka shows that collagen degradation also becomes the leading cause of AAA's deadly complication rupture. Increased collagen turnover has been reported in AAA in human beings, suggesting the existence of the repair process, as shown in animal model ${ }^{14}$.

Metabolic regulation of both elastin and collagen proteins in the aortic wall is under the control of several enzymatic 
agents. The most critical mediators appear to the zinc and MMPS ${ }^{8,15}$. Elastic and collagen fibers are degraded by proteolytic enzyme mostly represented by matrix metalloproteinases (MMP) locally activated either by other kin of MMP or by plasmin generated by plasminogen activators. There is evidence that abnormal local MMP production and regulation is associated with the pathogenesis of aortic aneurysm $^{16}$. The elastolytic subtypes MMP-9 and MMP-2 appear to be the most influential in AAA pathogenesis. Among the MMPs, the MMP-2 is unique in its ability to degrade both elastin and fibrillar collagen. It is found primarily in association with the mesenchymal cells of the media and adventitia, but small amounts may be produced by macrophages ${ }^{3,10}$. A study by Longo et al. showed that both total MMP-2 and activated MMP-2 are increased in AAA tissues. That study also hypothesized that mesenchymal cell, the MMP-2, was also involved in the initiation and progression of AAA. Higher plasma levels of MMP9, which have previously been shown to correlate with increased MMP-9 activity, have been associated with acute coronary syndrome, cerebrovascular disease, atherosclerosis, and AAA formation and rupture in several small studies ${ }^{17}$. A study by Gordin et al. showed that plasma levels of MMP-9 were independently associated with aortic wall thickness and aortic luminal diameter. In that study, MMP-9 activity is increased in the aortas of individuals with AAA compared to the non-diseased aorta, and areas of MMP-9 hyperactivity have been discovered in areas of aneurysm wall weakening and rupture $^{8,18}$. A study by $\mathrm{Yu}$ and Stamekovic showed that MMP-9 and MMP-2 could activate the TGF- $\beta$ by cleaving its latent form. Activation of TGF- $\beta$ plays a vital role in the control of tissue remodeling in physiology and pathologic situations. The mechanism by which alterations of TGF- $\beta$ promotes in AAA is currently controversial since the effects of blocking this cytokine vary in different models. The most detailed study to date suggests that at least in one model, TGF- $\beta$ protects against aortic aneurysm formation by inhibiting monocytemacrophage based tissue destruction via MMP-12 production ${ }^{17,19,20}$. The tissue inhibitors of matrix metalloproteinases (TIMP) are also increased in the wall of the aneurysm.

A chronic inflammatory infiltrate composed of $\mathrm{T}$ cells, macrophages, $\mathrm{B}$ lymphocytes, and plasma cells is a typical histologic feature in AAA. T cells, including both $\mathrm{CD} 4+$ and $\mathrm{CD} 8+$, are found in humans $\mathrm{AAAs}^{21}$. T cells found in both adventitia and media of human 
AAA biopsies. A high amount of $\mathrm{T}$ cells in human AAA may be caused by an increased influx or reduced clearance of these cells. Macrophages, neutrophils, and mast cells have been implicated in AAA formation ${ }^{22}$. Macrophages are demonstrated within the adventitia and media of human AAA biopsies. Human AAA biopsies contain significantly higher levels of neutrophils localized in the adventitia. Neutrophils recruitment to AAAS may be mediated by complement components $\mathrm{C} 3 \mathrm{a}$ and $\mathrm{C} 5 \mathrm{a}$ or neutrophilderived IL-8 and leukotriene B4. Antagonism of $\mathrm{C} 3 \mathrm{a}$ and $\mathrm{C} 5 \mathrm{a}$ blocked AAA formation in mouse models. Small numbers of mast cells are found in the media and adventitia of human AAA. The cell number has been correlated with AAA diameter. In rats, periaortic $\mathrm{CaCl} 2$ injury-induced AAA with the associated increase in mast cell content ${ }^{22,23}$.

Chlamydia pneumonia has been investigated to take a role in AAA. Chlamydia pneumonia infection has been related to more rapid AAA enlargement due to serological test evidence ${ }^{24}$. The development of AAA has associated with mural thrombus also. Thrombus is found in about $75 \%$ of all AAA (picture $1 \& 2$ ). Some studies showed that rupture has a correlation with thrombus in the aneurysm. There is also evidence that more massive AAA thrombus load is associated with a higher growth rate ${ }^{9,16}$. Evolution of aneurysmal diameter has been reported to correlate with plasma markers of fibrin formation and degradation as well as the circulating complex plasmin-a1-anti-plasmin potentially related to thrombus turnover ${ }^{25,26}$. The thrombus has been demonstrated to contain large numbers of neutrophils, MMPs, and cytokines. A study by Touat et al. showed that inhibition of platelet activation had been shown to slow AAA progression ${ }^{27}$. Although thrombus can reduce aneurysmal wall stress, its increasing thickness leads to local hypoxia at the inner layer of the media, which can induce increased medial neovascularization and inflammation ${ }^{9,26}$. The thrombus in aneurysmal enlargement in terms of a source of proteases has also been proposed after an initial report of enrichment of MMP-9 in the thrombus. A study by fountain et al. also showed the evidence op polymorphonuclear neutrophils trapping and storing MMP-9 in the aneurysmal thrombus. 


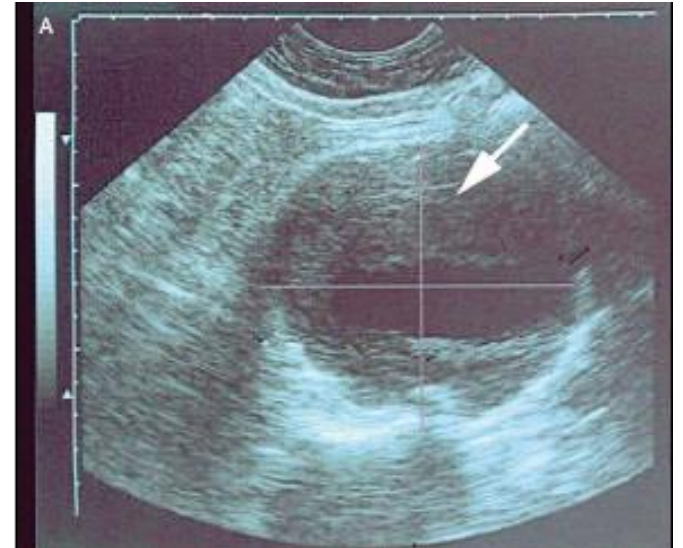

Picture 1. Sagittal section with parietal Thrombus in USG examination ${ }^{3}$

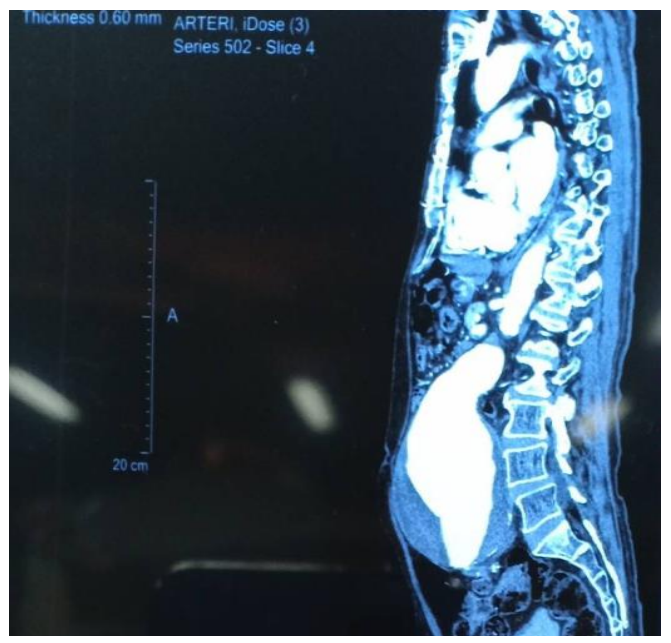

Picture 2. Thrombus in Multislice computed tomography (Source : $\quad$ Tahalele's documentation)

\section{CLINICAL PRESENTATION}

Unruptured Abdominal Aortic Aneurysm

The unruptured aneurysm is asymptomatic in most patients. Some of the patients are diagnosed incidentally by the clinician during the examination, especially in patients with coronary, peripheral, or cerebrovascular disease ${ }^{28}$. Chronic abdominal discomfort and back pain, which can result from direct pressure or distension of adjacent organs. In physical examination, an abdominal mass with pulsatile mass might be found. Auscultation of the abdomen reveals an intermittent bruit $^{29}$. The most important sign is the absence of hypovolemic shock symptoms suggesting that aneurysm has not ruptured. However, the recent onset of severe lumbar pain has been deemed to indicate the impending rupture. Rapid expansion $(>1 \mathrm{~cm} /$ year $)$ or the development of symptoms such as abdominal pain and tenderness and/or back pain is usually an indication for prompt surgical intervention, irrespective of size. This is because of high rupture rate $^{18}$

\section{Ruptured Abdominal Aortic Aorta}

Rupture of an aortic aneurysm is a sudden event with severe abdominal and/or back pain and circulatory collapse $^{30}$. Rupture of abdominal aortic aneurysm is heralded by a triad of sudden onset pain in the mid-abdomen or flank, shock, and the presence of a pulsatile abdominal mass. The degree of shock varies according to the location and size of the rupture. Frequently, rupture occurs into a retroperitoneal space, and bleeding may be arrested by a combination of hypotension and tamponade within this space. Most patients with rupture of the posterolateral wall into retroperitoneal 
space can reach the clinic alive. A small tear can temporarily seal the rupture, and the initial blood loss might be small. Free rupture into the peritoneal cavity is rapidly fatal. It is usually anterolateral rupture ${ }^{18,31}$. Approximately $75 \%$ of patients with an anterolateral rupture die before reaching the hospital. Very rarely, the aneurysm might spontaneously rupture into the duodenum. Rupture into the vena cava can take place with clinical symptom leg edema ${ }^{2,3}$.

\section{MANAGEMENT OF AAA}

\section{Principles of AAA Management}

Principle AAA management strategy is the prevention of rupture. Pharmacology therapy plays an important role consist of blood pressure control, cholesterol reduction, antiplatelet therapy, and smoking cessation ${ }^{32}$. Treatments with statins, angiotensinconverting enzyme inhibitors, antibiotics, and anti-inflammatory agents appear to inhibit the growth rate of AAA in humans. The size of AAA is still important to factor in the prediction of rupture. From a meta-analysis of 13 studies by Law et al., they already had quantified this annual risk for differing initial size $\mathrm{e}^{33}$.

\begin{tabular}{ll}
\hline $\begin{array}{l}\text { Maximum aortic } \\
\text { diameter }(\mathrm{cm})\end{array}$ & $\begin{array}{l}\text { Risk of } \\
\text { rupture }(\%)\end{array}$ \\
\hline & \\
3.0 & 0.0 \\
$3-3.9$ & 0.4 \\
$4-4.9$ & 1.1 \\
$5-5.9$ & 3.3 \\
$6-6.9$ & 9.4 \\
$7-7.9$ & 24.5 \\
\hline
\end{tabular}

Table 1. Size of AAA and Risk of Rupture ${ }^{33}$

\section{Open Surgical Treatment for AAA}

\section{Repair}

General anesthesia is preferred, and it is combined with epidural anesthesia to control the pain postoperatively. A broad-spectrum antibiotic administered as prophylaxis to prevent graft infection. An intravenous bolus of heparin should be given prior to clamp application ${ }^{2,34}$.

Aorta is a longitudinal midline structure, and it needs proximal and distal anastomoses in AAA operation. The abdomen is entered through a long midline or a transverse incision to get a transperitoneal approach. An oblique leftsided abdominal incision to get an extraperitoneal approach can be an alternative in selected patients. An extraperitoneal approach recommended in patients with chronic obstructive pulmonary disease. The disadvantages of this extraperitoneal approach are that intraperitoneal content can not be inspected, and it is often difficult to get the right iliac artery access. The aneurysm is exposed by incising the 
posterior parietal peritoneum and mobilizing the duodenum to the right side. Renal vein marks the upper limit for an infrarenal aneurysm. The neck of aneurysm needs to be identified to control it. Clamping on both common iliac arteries is needed, with care being taken to avoid damage to the hypogastric plexus of nerve in sexually active males. In some cases, encircling the inferior mesenteric artery with rubber to reimplant it on the aortic prosthesis needs to be done to maintain direct flow for sigmoid and rectum ${ }^{3}$.

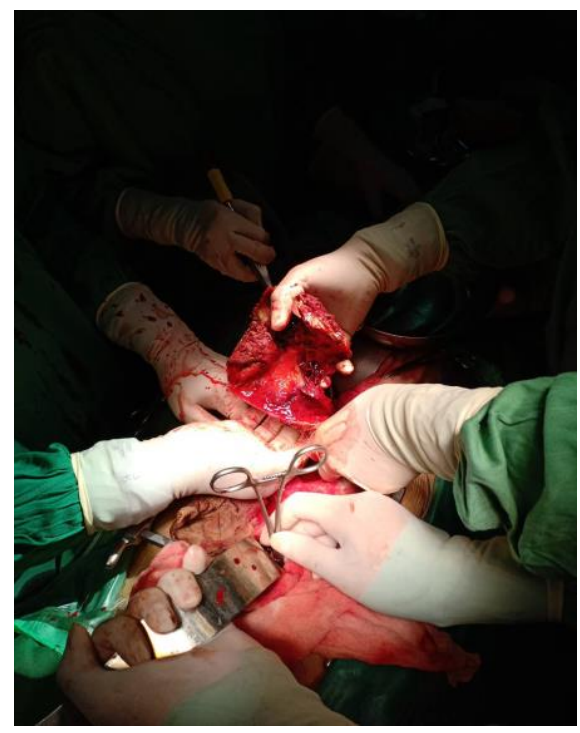

Fabric grafts constructed from coated polyester (Dacron) may be used. The upper anastomosis is of end to end type, and the distal anastomosis is located on the aortic bifurcation, the iliac bifurcation of the common femoral arteries depending on the extent of aneurysmal transformation. Iliac anastomoses should be preferred over femoral anastomoses because of the higher infection rate in groin anastomoses $^{35}$.

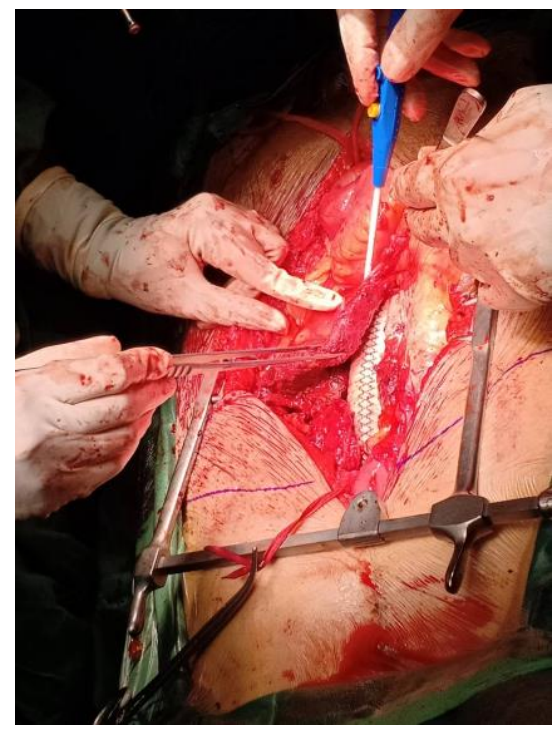

Picture 3. Thrombus extraction during AAA surgery (source : Tahalele's documentation). Picture 4. Dacron graft used for AAA repair (source: Tahalele's documentation) 


\section{Endovascular AAA Repair (EVAR)}

Parodi first introduced EVAR in 1991. This minimally invasive technique has become popular due to physicians and patients alike. Endovascular repair consists of the replacement of graft across the aneurysm and the fixation on the standard aortic wall and iliac wall with stents at both end $^{13,31}$. EVAR's goal is sustained aneurysm exclusion from the systemic circulation by means of a preoperative stent graft, preventing further aneurysm expansion and therefore eliminating further risk. The endograft of EVAR is composed of fabric and metal stents ${ }^{36}$. This stent is fed through iliac arteries by catheters and guidewires until the endograft is positioned correctly at the proximal and distal side of the aneurysmal segment ${ }^{37,38}$. The fixing device attaches to the aortic wall and hold the graft firmly in the place, excluding blood flow from the aneurysm sac and removing pressure from the aneurysm wall. Excellent results are characterized by perfectly canalized blood flow and retracted aneurysm wall around the endograft $^{35}$

\section{REFERENCE}

1. Kumar, Y. et al. Abdominal aortic aneurysm: Pictorial review of common appearances and complications. Ann. Transl. Med. 5, 1-7 (2017).

2. Aggarwal, S., Qamar, A., Sharma, V. \& Sharma, A. Abdominal aortic aneurysm: A comprehensive review. Exp. Clin. Cardiol. 16, 11-15 (2011).

3. Kahn, R. A. Abdominal aortic aneurysm. Clin. Cases Anesth. 195-199 (2005). doi:10.1016/B978-0-443-066245.50040-0

4. Lo, R. C. \& Schermerhorn, M. L. Abdominal aortic aneurysms in women. J. Vasc. Surg. 63, 839844 (2016).

5. Zommorodi, S., Leander, K., Roy, J., Steuer, J. \& Hultgren, R. Understanding abdominal aortic aneurysm epidemiology:

Socioeconomic position affects outcome. J. Epidemiol. Community Health 72, 904-910 (2018).

6. Kobeissi, E., Hibino, M., Pan, H. $\&$ Aune, D. Blood pressure, hypertension and the risk of abdominal aortic aneurysms: a systematic review and metaanalysis of cohort studies. Eur. J. Epidemiol. (2019). doi:10.1007/s10654-019-00510-9

7. Aune, D., Schlesinger, S., Norat, T. \& Riboli, E. Tobacco smoking and the risk of abdominal aortic aneurysm: a systematic review and meta-analysis of prospective studies. Sci. Rep. 8, 1-9 (2018).

8. Abdul-Hussien, H. et al. Collagen degradation in the abdominal aneurysm: A conspiracy of matrix metalloproteinase and cysteine collagenases. Am. J. Pathol. 170, 809-817 (2007).

9. Vorp, D. A. Biomechanics of abdominal aortic aneurysm. $J$. Biomech. 40, 1887-1902 (2007).

10. Maguire, E. M., Pearce, S. W. A., Xiao, R., Oo, A. Y. \& Xiao, Q. Matrix metalloproteinase in abdominal aortic aneurysm and aortic dissection. Pharmaceuticals 12, 1-18 (2019).

11. Khera, A., Mcguire, D. K., Lemos, J. A. De \& Das, S. R. aortic 
pathology: From the Dallas Heart Study. 16, 339-345 (2012).

12. Kuivaniemi, H., Ryer, E. J., Elmore, J. R. \& Tromp, G. Understanding the pathogenesis of abdominal aortic aneurysms. Expert Rev. Cardiovasc. Ther. 13, 975-987 (2015).

13. Moll, F. L. et al. Management of abdominal aortic aneurysms clinical practice guidelines of the European society for vascular surgery. Eur. J. Vasc. Endovasc. Surg. 41, (2011).

14. Dobrin, P. B., Mrkvicka, R. \& Maywood, D. L. Failure ofelastin or collagen aspossible critical connectivetissuealterations underlying aneurysmal dilatation. 2, 484-488

15. Nackman, G. B., Karkowski, F. J., Halpern, V. J., Gaetz, H. P. \& Tilson, M. D. Elastin degradation products induce adventitial angiogenesis in the Anidjar/Dobrin rat aneurysm model. Surgery 122, 39-44 (1997).

16. Dobrin, P. B. Pathophysiology and pathogenesis of aortic aneurysms. Current concepts. Surg. Clin. North Am. 69, 687-703 (1989).

17. Longo, G. M. et al. Work in Concert To Produce Aortic Aneurysms. J. Clin. Invest. 110, 625-632 (2002).

18. Badger, S. et al. Endovascular treatment for ruptured abdominal aortic aneurysm. Cochrane Database Syst. Rev. 2017, (2017).

19. Wang, Y., Krishna, S., Walker, P. J., Norman, P. \& Golledge, J.

Transforming growth factor- $\beta$ and abdominal aortic aneurysms. Cardiovasc. Pathol. 22, 126-132 (2013).

20. Thatcher, S. E. TGF- $\beta$ Signaling:

New Insights Into Aortic Aneurysms. EBioMedicine 12, 24 25 (2016).

21. Li, H. et al. Modulation of immune-inflammatory responses in abdominal aortic aneurysm: Emerging molecular targets. $J$. Immunol. Res. 2018, (2018).

22. Shimizu, K., Mitchell, R. N. \& Libby, P. Inflammation and cellular immune responses in abdominal aortic aneurysms. Arterioscler. Thromb. Vasc. Biol. 26, 987-994 (2006).

23. Lindholt, J. S. \& Shi, G. P. Chronic Inflammation, Immune Response, and Infection in Abdominal Aortic Aneurysms. Eur. J. Vasc. Endovasc. Surg. 31, 453-463 (2006).

24. Edvinsson, M., Thelin, S., Hjelm, E., Friman, G. \& NyströmRosander, C. Persistent Chlamydophila pneumoniae infection in thoracic aortic aneurysm and aortic dissection? Ups. J. Med. Sci. 115, 181-186 (2010).

25. Haller, S. J. et al. Intraluminal thrombus is associated with early rupture of abdominal aortic aneurysm. J. Vasc. Surg. 67, 10511058.e1 (2018).

26. Piechota-Polanczyk, A. et al. The Abdominal Aortic Aneurysm and Intraluminal Thrombus: Current Concepts of Development and Treatment. Front. Cardiovasc. Med. 2, 1-14 (2015).

27. Touat, Z. et al. Renewal of mural thrombus releases plasma markers and is involved in aortic abdominal aneurysm evolution. Am. J. Pathol. 168, 1022-1030 (2006).

28. Patel, H. et al. Thrombosis of abdominal aortic aneurysms. Am. Surg. 60, 801-803 (1994).

29. Petriceks, A. H., Olivas, J. C. \& Salmi, D. Educational Case: Symptomatic but Unruptured Abdominal Aortic Aneurysm. Acad. Pathol. 5, 237428951879856 (2018). 
30. Thompson, M. M., Morgan, R. A., Matsumura, J. S., Sapoval, M. \& Loftus, I. M. Endovascular Intervention for Vascular Disease. (Informa, 2008).

31. Chaikof, E. L. et al. The Society for Vascular Surgery practice guidelines on the care of patients with an abdominal aortic aneurysm. J. Vasc. Surg. 67, 277.e2 (2018).

32. Miyake, T. \& Morishita, R. Pharmacological treatment of abdominal aortic aneurysm. Cardiovasc. Res. 83, 436-443 (2009).

33. R, L. M., J, M. \& Wald, N. Screening for Abdominal Aortic Aneurysms. Vasc. Diagnosis 381387 (2005). doi:10.1016/B978-07216-9426-9.50042-X

34. Schermerhorn, M. L. et al. Endovascular vs. open repair of abdominal aortic aneurysms in the medicare population. $N$. Engl. $J$. Med. 358, 464-474 (2008).

35. Parodi, J. C., Palmaz, J. C. \& Barone, H. D. Transfemoral Intraluminal Graft Implantation for Abdominal Aortic Aneurysms. Ann. Vasc. Surg. 5, 491-499 (1991).

36. Prinssen, M. et al. A randomized trial comparing conventional and endovascular repair of abdominal aortic aneurysms. N. Engl. J. Med. 351, 1607-1618 (2004).

37. D'sa, A. A. B. \& Chant, A. D.

Emergency Vascular and Endovascular Surgical Practice. (Hodder Arnold, 2005).

38. Blankensteijn, J. D. et al. Twoyear outcomes after conventional or endovascular repair of abdominal aortic aneurysms. $N$. Engl. J. Med. 352, 2398-2405 (2005). 